\title{
Comparison between the Effects of Insulin versus Corticosteroid Local Injections in Diabetic Patients with Carpal Tunnel Syndrome
}

Ahmed Ismail Ali Shalaby ${ }^{1}$ Kalthom Mohamed Abd El Hamid ${ }^{1}$, Ibrahim Abd Alsamie Emam ${ }^{1}$, Adel Ibrahim Azzam ${ }^{1}$, Mohamed Ahmed Zaki ${ }^{2}$

\author{
${ }^{1}$ Department of Physical Medicine, Rheumatology and Rehabilitation, ${ }^{2}$ Department of \\ Neurology, Faculty of Medicine, Al-Azhar University, Cairo, Egypt
}

Corresponding author: Ahmed Ismail Ali Shalaby; Mobile: 01145513426; Email: dr.ahmedismail. rheumatologist@gmail.com

\begin{abstract}
Background: Carpal tunnel syndrome (CTS) is a medical condition due to compression of the median nerve as it travels through the wrist at the carpal tunnel. Carpal tunnel syndrome is the most commonly diagnosed and treated entrapment neuropathy and is a significant cause of morbidity. It is estimated to affect one out of ten people during their lifetime. Diabetic patients have a higher incidence with carpal tunnel syndrome. It has been reported that insulin improves the nerve regeneration and myelination through its role as neurotrophic factor.
\end{abstract}

Aim of the Work: was to compare the short-term effects of insulin and corticosteroid local injections in the treatment of diabetic patients with carpal tunnel syndrome.

Patients and Methods: Fifty diabetic patients with clinical and electrophysiological evidence of mild to moderate carpal tunnel syndrome were included in this study. All Patients had been sub-classified into two equal groups:- Group (1): Twenty five patients have received local insulin injection of $10 \mathrm{IU}$ Neutral Protamine Hagedorn (NPH) \{Insulinagypt N\} into the affected carpal tunnel at the first visit and the same dose of insulin after 2 weeks. Group (2): twenty five patients have received a single dose of $40 \mathrm{mg}$ triamcinolone acetonide \{Epirelefan\} local injection into the affected carpal tunnel. Nerve conduction study (NCS), Boston Carpal Tunnel Questionnaire (BCTQ) and clinical evaluation were carried out for both groups at baseline and four weeks after second dose of insulin injection and one month after steroid injection.

Results: In steroid group, significant improvement in nerve conduction study as regard distal sensory latency and sensory conduction velocity without significant improvement in distal motor latency and motor conduction velocity and significant improvement in (BCTQ). In insulin group, a more significant improvement in all parameters of nerve conduction study and also in (BCTQ) was observed with insulin injection than steroid done. Also, there were significant differences between results of both groups for insulin injection group.

Conclusion: Local insulin injection is an effective treatment for restoring median nerve function in diabetic patients with mild to moderate carpal tunnel syndrome and produce significant improvement in NCS and BCTQ.

Keywords: Carpal tunnel syndrome, diabetes, Insulin, steroid, local injection.

\section{INTRODUCTION}

Carpal tunnel syndrome (CTS) is a medical condition due to compression of the median nerve as it travels through the wrist at the carpal tunnel ${ }^{[1]}$ and it is the most commonly diagnosed and treated entrapment neuropathy and is a significant cause of morbidity. The estimated prevalence of CTS in general population is 1 to 5 percent ${ }^{[2]}$. CTS is more frequent in women ( 0.7 to 9.2 percent) than in men $(0.4 \text { to } 2.1 \text { percent })^{[3]}$. CTS is reported to occur in $2.6-20 \%$ of all patients with diabetes [4].
The main symptoms are pain, numbness and tingling in the thumb, index finger, middle finger and the thumb side of the ring fingers ${ }^{[1]}$, which occurs following entrapment of the median nerve within the carpal tunnel ${ }^{[5]}$. Symptoms typically start gradually and during the night, Pain may extend up the arm, on awakening, the patient usually gets relief by shaking or rubbing the hands. Weak grip strength may occur and after a long period of time the muscles at the base of the thumb may waste away. In more than half of cases both sides are affected ${ }^{[1]}$. Diagnosis is suspected 
based on signs, symptoms, and specific physical tests and may be confirmed with electro-diagnostic study ${ }^{[6]}$.

Symptoms can be improved by wearing a wrist splint or with corticosteroid injections. Taking NSAIDs or gabapentin does not appear to be useful. Surgery to release the transverse carpal ligament is effective with better results at a year compared to non- surgical options. Further splinting after surgery is not needed ${ }^{[6]}$.

It is accepted that chronic increase in pressure within the carpal tunnel is responsible for median nerve ischemia and subsequent segmental demyelination ${ }^{[7]}$. It has been assumed that hyperglycemia induces metabolic derangement that directly affect Schwann cells, nodes of Ranvier and that hyperglycemia and metabolic derangement affect the structure and function of endoneurial microvessels ${ }^{[8]}$.

It has been reported that peripheral nerves carry abundant receptors for nerve growth factor (NGF), a member of the insulin like growth factor-1 (IGF-1) family, and insulin [9]. Both of these are thought to promote neuronal growth and regeneration and could be important in restoring nerve function following metabolic or vascular damage. There have been several reports assessing the effects of NGF on diabetic peripheral neuropathy and its effect on nerve regeneration ${ }^{[10]}$. In their study, Singhal et al. ${ }^{[11]}$ found out that near-nerve local insulin injection prevented conduction slowing in experimental diabetes.

The aim of this work was to evaluate the effect of local insulin injection in improvement of median nerve function in diabetic patients with mild to moderate CTS in comparison to local steroid injection.

\section{PATIENTS AND METHODS}

This study included a total of fifty diabetic patients their age ranged from 30 to 60 years (35 females and 15 males) with clinical and electrophysiological evidence of mild to moderate CTS according to Bland's classification ${ }^{[12]}$, who were recruited from Physical Medicine, Rheumatology and Rehabilitation Outpatient Clinic at Al-Azher University Hospitals. Approval of the Ethical Research Committee of the Faculty of Medicine, Al-Azhar University and a written informed consent from all the subjects were obtained. This study was conducted between February 2018 to July 2018.

Inclusion criteria: All diabetic patients with mild to moderate CTS aged from 30 to 60 years diagnosed by electrophysiological studies.

Exclusion criteria: Those patients with one of the following features were excluded from this study: Thenar atrophy, previous carpal tunnel release surgery, previous local injection, pregnancy, hypothyroidism, polyneuropathy and rheumatoid arthritis.

All patients were screened for the presence of mild to moderate carpal tunnel syndrome. Screening was based on symptoms, Boston Carpal Tunnel Questionnaire (BCTQ) and clinical examination as well as by nerve conduction study.

Patients had been sub-classified into two equal groups: Group (1): Twenty five patients with carpal tunnel syndrome have received local insulin injection of $10 \mathrm{IU}$ $\mathrm{NPH}$ \{Insulinagypt $\mathrm{N}\}$ into the affected carpal tunnel at the first visit and the same dose of insulin after 2 weeks. Group (2):- twenty five patients with carpal tunnel syndrome have received a single dose of $40 \mathrm{mg}$ triamcinolone acetonide $\{$ Epirelefan local injection into the affected carpal tunnel.

\section{All patients had been subjected for the following at base line:}

1. Complete history taking including: age, sex, disease duration, diabetes mellitus duration, paresthesia in the fingers innervated by median nerve, severity of hand pain especially at night and wasting in the thenar muscles.

2. Clinical examination including: sensory and motor examination of the hand, and special tests for CTS (Tinel's test \& Phalen test).

3. Anthropometric measurements including: Weight, Height and Body mass index (BMI) was calculated as the weight (in $\mathrm{kg}$ ) divided by height (in $\mathrm{m}^{2}$ ).

4. Laboratory Investigations including: Fasting Blood Sugar (FBS), Rheumatoid Factor (RF) and Thyroid Stimulating Hormone (TSH). 
All patients had been subjected for the following at base line and four weeks after second dose of insulin injection and one month after steroid injection:

5. Assessment of clinical severity of CTS by: Boston Carpal Tunnel Questionnaire (BCTQ), which has two component :- Symptom severity by symptom severity scale (SSS) and Functional disability by functional status scale (FSS). BCTQ is a diseasespecific measure of self-reported symptom severity and functional status for CTS. BCTQ is a valid, reliable, responsive and acceptable instrument to measures CTS outcome ${ }^{[13]}$.The SSS consist of 11 questions, the FSS consist of 8 questions and both use a five-point scale. Each scale generates a final score (sum of individual item scores divided by the number of items) that ranges from 1 to 5 with a higher score indicating greater disability.

6. Electrophysiological assessment: were performed by an EMG/NCS unit: - NCSs record was done by using Nihon Kohden Neuropack MEB2300J/K, EMG/NCV/EP Measuring Desktop System- 6 spaces channels Digital, Japan. The used electric stimulator, the surface electrodes and the ground electrode used were all Nihon Kohden Neuropack, Japan.

Procedures of nerve conduction study: Motor nerve conduction study of median nerve: by applying recording electrode (active electrode) on motor point of the abductor pollicis brevis muscle, Reference electrode $3 \mathrm{~cm}$ distal to active electrode and Stimulating sites were at the wrist and at the elbow in the antecubital fossa.

\section{Anti dromic sensory nerve conduction study of median nerve done by using ring electrode: by applying active electrode on middle phalanx of index finger, Reference electrode on distal phalanx of index finger and Stimulating site were at the wrist and with $14 \mathrm{~cm}$ from recording electrodes.}

\footnotetext{
Motor nerve conduction study of ulnar nerve: by applying recording electrode (active electrode) on motor point of the abductor digiti minimi muscle, Reference electrode $3 \mathrm{~cm}$ distal to active electrode and Stimulating sites were at
}

the wrist and at the elbow posterior to the medial epicondyle of the humerus.

Anti dromic sensory nerve conduction study of ulnar nerve done by using ring electrode: by applying active electrode on middle phalanx of little finger, Reference electrode on distal phalanx of little finger and Stimulating site were at the wrist and with $14 \mathrm{~cm}$ from recording electrodes.

The patient with abnormal sensory and normal motor distal latencies of median nerve (prolonged antidromic distal sensory latency $>3.5 \mathrm{~ms}$ ) considered as mild carpal tunnel syndrome ${ }^{[12]}$. The patient with abnormal sensory and motor distal latencies of median nerve (prolonged distal motor latency $>4.2 \mathrm{~ms}$ but $<6.5 \mathrm{~ms}$ and prolonged antidromic distal sensory latency $>3.5 \mathrm{~ms}$ ) considered as moderate CTS ${ }^{[12]}$.

Technique of injection: Proximal wrist crease (or $1 \mathrm{~cm}$ proximal to most distal wrist crease) had been marked and ulnar side ( about $1 / 2 \mathrm{~cm}$ ) from palmaris longus or wrist midline if palmaris longus absent, this is the site of injection. Needle is inserted about 30-45 degrees distally toward middle-ring finger ${ }^{[14]}$. During the period of the study, No any drugs or physical therapy allowed to be taken.

Statistical analysis: Statistical presentation and analysis of the present study was conducted using the mean, standard deviation and chisquare test by Statistical Package for Social Science version 22 (SPSS V.22). Qualitative data were presented as number and percentages while quantitative data with parametric distribution were presented as mean, standard deviations and ranges. The $\mathrm{p}$-value, $\mathrm{P}<$ 0.05 was considered as significant and $\mathrm{P}>$ 0.05 was considered as non-significant.

\section{RESULTS}

50 diabetic patients were included in this study, patients with bilateral CTS, the most symptomatic hand was selected, so the total hands here were 50 hands.

Table [1]:- Demographic, clinical characteristics and Laboratory investigations in both groups.

As regard age, the mean age of patients in insulin group was $46.28 \pm 8.48$ years while in steroid group was $46.00 \pm 8.28$ years. As regard 
weight, the mean weight of patients in insulin group was $79.84 \pm 11.89 \mathrm{~kg}$ while in steroid group was $80.28 \pm 11.19 \mathrm{~kg}$. As regard height, the mean height of patients in insulin group was $162.60 \pm 7.34 \mathrm{~cm}$ while in steroid group was $163.96 \pm 6.82 \mathrm{~cm}$. As regard BMI, the mean BMI of patients in insulin group was $30.16 \pm 4.10 \mathrm{~kg} / \mathrm{m}^{2}$ while in steroid group was $29.91 \pm 4.35 \mathrm{~kg} / \mathrm{m}^{2}$. As regard DM duration, the mean DM duration of patients in insulin group was $5.36 \pm 1.11$ years while in steroid group was $5.8 \pm 1.29$ years. As regard CTS duration, the mean CTS duration of patients in insulin group was $10.24 \pm 6.86$ weeks while in steroid group was $15.24 \pm 11.30$ weeks. As regard FBS, the mean FBS of patients in insulin group was $132.40 \pm 30.46 \mathrm{mg} / \mathrm{dl}$ while in steroid group was $136.12 \pm 30.58 \mathrm{mg} / \mathrm{dl}$. As regard TSH, the mean TSH of patients in insulin group was $1.98 \pm 0.98$ $\mathrm{uIU} / \mathrm{ml}$ while in steroid group was $2.15 \pm 0.69 \mathrm{uIU} / \mathrm{ml}$. As regard RF, the RF in both groups were $<6 \mathrm{IU} / \mathrm{ml}$ (negative).

\begin{tabular}{|c|c|c|c|c|c|c|}
\hline & & Mean & & S. D & $\begin{array}{l}\text { t. } \\
\text { test }\end{array}$ & $\begin{array}{c}\mathrm{p} . \\
\text { valu } \\
\mathrm{e}\end{array}$ \\
\hline \multirow{2}{*}{$\begin{array}{c}\text { Age } \\
\text { (years) }\end{array}$} & $\begin{array}{c}\text { Insuli } \\
n\end{array}$ & 46.28 & \pm & 8.48 & \multirow{2}{*}{$\begin{array}{l}0.01 \\
4\end{array}$} & \multirow{2}{*}{$\begin{array}{l}0.90 \\
6\end{array}$} \\
\hline & $\begin{array}{c}\text { Steroi } \\
\mathrm{d}\end{array}$ & 46.00 & \pm & 8.28 & & \\
\hline \multirow{2}{*}{$\begin{array}{l}\text { Weight } \\
\text { (kg) }\end{array}$} & $\begin{array}{c}\text { Insuli } \\
n\end{array}$ & 79.84 & \pm & $\begin{array}{c}11.8 \\
9\end{array}$ & \multirow{2}{*}{$\begin{array}{l}0.01 \\
8\end{array}$} & \multirow{2}{*}{$\begin{array}{l}0.89 \\
3\end{array}$} \\
\hline & $\begin{array}{c}\text { Steroi } \\
\text { d }\end{array}$ & 80.28 & & $\begin{array}{c}11.1 \\
9\end{array}$ & & \\
\hline \multirow{2}{*}{$\begin{array}{l}\text { Height } \\
(\mathrm{cm})\end{array}$} & $\begin{array}{c}\text { Insuli } \\
n\end{array}$ & $\begin{array}{c}162.6 \\
0\end{array}$ & \pm & 7.34 & \multirow{2}{*}{$\begin{array}{l}0.46 \\
1\end{array}$} & \multirow{2}{*}{$\begin{array}{l}0.50 \\
1\end{array}$} \\
\hline & $\begin{array}{c}\text { Steroi } \\
\text { d }\end{array}$ & $\begin{array}{c}163.9 \\
6\end{array}$ & \pm & 6.82 & & \\
\hline \multirow{2}{*}{$\begin{array}{c}\mathrm{BMI} \\
\left(\mathrm{Kg} / \mathrm{m}^{2}\right)\end{array}$} & $\begin{array}{c}\text { Insuli } \\
n\end{array}$ & 30.16 & \pm & 4.10 & \multirow{2}{*}{$\begin{array}{l}0.04 \\
3\end{array}$} & \multirow{2}{*}{$\begin{array}{l}0.83 \\
6\end{array}$} \\
\hline & $\begin{array}{c}\text { Steroi } \\
\mathrm{d}\end{array}$ & 29.91 & \pm & 4.35 & & \\
\hline \multirow{2}{*}{$\begin{array}{c}\mathrm{DM} \\
\text { duratio } \\
\mathrm{n} \\
\text { (years) }\end{array}$} & $\begin{array}{c}\text { Insuli } \\
n\end{array}$ & 5.36 & \pm & 1.11 & \multirow{2}{*}{$\begin{array}{l}1.66 \\
5\end{array}$} & \multirow{2}{*}{$\begin{array}{l}0.20 \\
3\end{array}$} \\
\hline & $\begin{array}{c}\text { Steroi } \\
\mathrm{d}\end{array}$ & 5.8 & \pm & 1.29 & & \\
\hline \multirow{2}{*}{$\begin{array}{c}\text { CTS } \\
\text { duratio } \\
n \\
\text { (weaks) }\end{array}$} & $\begin{array}{c}\text { Insuli } \\
n\end{array}$ & 10.24 & \pm & 6.86 & \multirow{2}{*}{$\begin{array}{l}3.57 \\
9\end{array}$} & \multirow{2}{*}{$\begin{array}{l}0.06 \\
5\end{array}$} \\
\hline & $\begin{array}{c}\text { Steroi } \\
\mathrm{d}\end{array}$ & 15.24 & \pm & $\begin{array}{c}11.3 \\
0\end{array}$ & & \\
\hline \multirow{2}{*}{$\begin{array}{c}\text { FBS } \\
(\mathrm{mg} / \mathrm{dl})\end{array}$} & $\begin{array}{c}\text { Insuli } \\
n\end{array}$ & $\begin{array}{c}132.4 \\
0\end{array}$ & \pm & $\begin{array}{c}30.4 \\
6 \\
\end{array}$ & \multirow{2}{*}{$\begin{array}{l}0.18 \\
6\end{array}$} & \multirow{2}{*}{$\begin{array}{l}0.66 \\
8\end{array}$} \\
\hline & $\begin{array}{c}\text { Steroi } \\
\mathrm{d}\end{array}$ & $\begin{array}{c}136.1 \\
2 \\
\end{array}$ & \pm & $\begin{array}{c}30.5 \\
8 \\
\end{array}$ & & \\
\hline \multirow{2}{*}{$\begin{array}{c}\text { TSH } \\
(\mathrm{uIU} / \mathrm{ml} \\
)\end{array}$} & $\begin{array}{c}\text { Insuli } \\
\mathrm{n}\end{array}$ & 1.98 & \pm & 0.98 & \multirow{2}{*}{$\begin{array}{l}0.50 \\
8\end{array}$} & \multirow{2}{*}{$\begin{array}{l}0.47 \\
9\end{array}$} \\
\hline & $\begin{array}{c}\text { Steroi } \\
\mathrm{d}\end{array}$ & 2.15 & \pm & 0.69 & & \\
\hline
\end{tabular}

\begin{tabular}{|c|c|ll|}
\hline \multirow{2}{*}{$\mathrm{RF}$} & Insuli & $<6$ & \\
$\mathrm{n}$ & $\mathrm{IU} / \mathrm{ml})$ & Negative \\
\cline { 2 - 4 } & $\begin{array}{c}\text { Steroi } \\
\mathrm{d}\end{array}$ & $<6$ & Negative \\
\hline
\end{tabular}

S.D: standard deviation DM: diabetes mellitus.

Table 1, shows that there was no statistical significant difference between insulin and steroid locally injected groups regarding Age, Weight, Height, BMI, DM duration, CTS duration, Fasting Blood Sugar, TSH (p-value $>0.05$ ) and RF.

Table [2]:- Male and Female distribution for insulin and steroid locally injected groups.

As regard sex, patients in insulin group was $24.0 \%$ males and $76.0 \%$ females while in steroid group was $36.0 \%$ male and $64.0 \%$ female with total patients in whole study were $30 \%$ males and $70 \%$ females.

\begin{tabular}{|c|c|c|c|c|c|}
\hline \multicolumn{3}{|l|}{ Sex } & $\begin{array}{c}\text { Insuli } \\
n\end{array}$ & $\begin{array}{c}\text { Steroi } \\
\mathrm{d}\end{array}$ & Total \\
\hline \multirow{2}{*}{\multicolumn{2}{|c|}{ Male }} & $\mathbf{N}$ & 6 & 9 & 15 \\
\hline & & $\%$ & $24.0 \%$ & $36.0 \%$ & $30.0 \%$ \\
\hline \multirow{2}{*}{\multicolumn{2}{|c|}{ Female }} & $\mathbf{N}$ & 19 & 16 & 35 \\
\hline & & $\%$ & $76.0 \%$ & $64.0 \%$ & $70.0 \%$ \\
\hline \multirow{2}{*}{\multicolumn{2}{|c|}{ Total }} & $\mathbf{N}$ & 25 & 25 & 50 \\
\hline & & $\%$ & $\begin{array}{c}100.0 \\
\%\end{array}$ & $\begin{array}{c}100.0 \\
\%\end{array}$ & $\begin{array}{c}100.0 \\
\%\end{array}$ \\
\hline \multirow[b]{2}{*}{$\begin{array}{l}\text { Chi- } \\
\text { square }\end{array}$} & $\mathbf{X}^{2}$ & \multicolumn{4}{|c|}{0.857} \\
\hline & $\begin{array}{c}\text { P- } \\
\text { valu } \\
\text { e }\end{array}$ & \multicolumn{4}{|c|}{0.355} \\
\hline
\end{tabular}

$\mathrm{X}^{2}$ : Chi-square test. $\mathrm{N}:$ Number.

Table 2, shows that there was no statistical significant difference between insulin and steroid locally injected groups regarding number and percentage of male and female in both groups and total number and percentage of male and female in the whole study ( $\mathrm{p}$-value $>0.05$ ).

Table [3]:- Classification of severity for CTS among Insulin and Steroid groups.

As regard severity of CTS, patients in insulin group considered as $60.0 \%$ mild and $40.0 \%$ moderate CTS while in steroid group was $52.0 \%$ mild and $48.0 \%$ moderate CTS with total patients in whole study were $56.0 \%$ mild and $44.0 \%$ moderate CTS.

\begin{tabular}{|c|c|c|c|c|}
\hline \multicolumn{2}{|c|}{ Severity Of CTS } & Insulin & Steroid & Total \\
\hline \multirow{2}{*}{ Mild } & $\mathbf{N}$ & 15 & 13 & 28 \\
\cline { 2 - 5 } & $\mathbf{\%}$ & $60.0 \%$ & $52.0 \%$ & $56.0 \%$ \\
\hline \multirow{2}{*}{ Moderate } & $\mathbf{N}$ & 10 & 12 & 22 \\
\cline { 2 - 5 }
\end{tabular}




\begin{tabular}{|c|c|c|c|c|c|}
\hline \multirow{3}{*}{\multicolumn{2}{|c|}{ Total }} & $\%$ & $40.0 \%$ & $48.0 \%$ & $44.0 \%$ \\
\hline & & $\mathbf{N}$ & 25 & 25 & 50 \\
\hline & & $\%$ & $100.0 \%$ & $100.0 \%$ & $100.0 \%$ \\
\hline \multirow{2}{*}{$\begin{array}{l}\text { Chi- } \\
\text { square }\end{array}$} & $\mathrm{X}^{2}$ & \multicolumn{4}{|c|}{0.322} \\
\hline & $\begin{array}{c}\text { P- } \\
\text { value }\end{array}$ & \multicolumn{4}{|c|}{0.569} \\
\hline
\end{tabular}

Table 3, shows that there was no statistical significant difference between insulin and steroid locally injected groups regarding number and percentage of mild and moderate degree of CTS in both groups and total number of mild and moderate degree of CTS and their percentage in the whole study ( $\mathrm{p}$-value >0.05).

Table [4]:-Boston Carpal Tunnel Questionnaire and Electrophysiological finding before and after steroid injection.

As regard BCTQ in steroid group, the mean SSS before injection was $2.87 \pm 0.47$ while after injection was $1.92 \pm 0.44$. The mean FSS before injection was $2.17 \pm 0.52$ while after injection was $1.64 \pm 0.38$. As regard NCS in steroid group, the mean DML before injection was4.60 \pm 0.89 while after injection was $4.32 \pm 0.91$. The mean DSL before injection was $3.97 \pm 0.42$ while after injection was $3.72 \pm 0.45$. The mean MCV before injection was $52.65 \pm 4.22$ while after injection was 54.26 \pm 4.47 . The mean SCV before injection was $36.16 \pm 3.75$ while after injection was $39.45 \pm 4.91$.

\begin{tabular}{|c|c|c|c|c|c|c|}
\hline & & $\begin{array}{c}\text { Mea } \\
\mathrm{n}\end{array}$ & \pm & $\begin{array}{l}\text { S. } \\
\text { D }\end{array}$ & t. test & $\begin{array}{c}\mathrm{p} . \\
\text { value }\end{array}$ \\
\hline \multirow{2}{*}{$\begin{array}{c}\text { BCTQ } \\
\text { (SSS) } \\
\text { Steroi } \\
\text { d }\end{array}$} & $\begin{array}{c}\text { Befor } \\
\text { e }\end{array}$ & 2.87 & \pm & $\begin{array}{c}0.4 \\
7\end{array}$ & \multirow{2}{*}{$\begin{array}{c}54.39 \\
5\end{array}$} & \multirow{2}{*}{$\begin{array}{c}0.001 \\
*\end{array}$} \\
\hline & After & 1.92 & \pm & $\begin{array}{c}0.4 \\
4\end{array}$ & & \\
\hline \multirow{2}{*}{$\begin{array}{c}\text { BCTQ } \\
\text { (FSS) } \\
\text { Steroi } \\
\text { d }\end{array}$} & $\begin{array}{c}\text { Befor } \\
\mathrm{e}\end{array}$ & 2.17 & \pm & $\begin{array}{c}0.5 \\
2\end{array}$ & \multirow[b]{2}{*}{$\begin{array}{c}16.85 \\
1\end{array}$} & \multirow[b]{2}{*}{$\begin{array}{c}0.001 \\
*\end{array}$} \\
\hline & After & 1.64 & \pm & $\begin{array}{c}2 \\
0.3 \\
8\end{array}$ & & \\
\hline \multirow{2}{*}{$\begin{array}{c}\text { DML } \\
\text { Steroi } \\
\text { d }\end{array}$} & $\begin{array}{c}\text { Befor } \\
\mathrm{e}\end{array}$ & 4.60 & \pm & $\begin{array}{c}0.8 \\
9\end{array}$ & \multirow{2}{*}{1.251} & \multirow{2}{*}{0.269} \\
\hline & After & 4.32 & \pm & $\begin{array}{c}0.9 \\
1\end{array}$ & & \\
\hline \multirow{2}{*}{$\begin{array}{c}\text { DSL } \\
\text { Steroi } \\
\text { d }\end{array}$} & $\begin{array}{c}\text { Befor } \\
\text { e }\end{array}$ & 3.97 & \pm & $\begin{array}{c}0.4 \\
2\end{array}$ & \multirow{2}{*}{4.183} & \multirow{2}{*}{$\begin{array}{c}0.046 \\
*\end{array}$} \\
\hline & After & 3.72 & \pm & $\begin{array}{c}0.4 \\
5\end{array}$ & & \\
\hline \multirow{2}{*}{$\begin{array}{c}\text { MCV } \\
\text { Steroi } \\
\text { d }\end{array}$} & $\begin{array}{c}\text { Befor } \\
\text { e }\end{array}$ & $\begin{array}{c}52.6 \\
5\end{array}$ & \pm & $\begin{array}{c}4.2 \\
2\end{array}$ & \multirow{2}{*}{1.710} & \multirow{2}{*}{0.197} \\
\hline & After & $\begin{array}{c}54.2 \\
6\end{array}$ & \pm & $\begin{array}{c}4.4 \\
7\end{array}$ & & \\
\hline
\end{tabular}

\begin{tabular}{|c|c|c|c|c|c|c|}
\hline \multirow{2}{*}{$\begin{array}{c}\text { SCV } \\
\text { Steroi } \\
\text { d }\end{array}$} & $\begin{array}{c}\text { Befor } \\
\text { e }\end{array}$ & $\begin{array}{c}36.1 \\
6\end{array}$ & \pm & $\begin{array}{c}3.7 \\
5\end{array}$ & \multirow{2}{*}{7.096} & \multirow{2}{*}{$\begin{array}{c}0.001 \\
*\end{array}$} \\
\hline & After & $\begin{array}{c}39.4 \\
5\end{array}$ & \pm & $\begin{array}{c}4.9 \\
1\end{array}$ & & \\
\hline
\end{tabular}

DML: distal motor latency. DSL: distal sensory latency. MCV: motor conduction velocity. SCV: sensory conduction velocity.

Table 4, shows that there was statistical significant difference in BCTQ as regard SSS and FSS (p-value $=0.001$ ) and also show statistical significant difference in some of NCS parameters as regard DSL and SCV (Pvalue $<0.05$ and $=0.001$ respectively) and there was no statistical significant difference as regard DML and MCV ( $p$-value>0.05), before and one month after steroid injection.

Table [5]:-Boston Carpal Tunnel Questionnaire and Electrophysiological finding before and after insulin injection.

As regard BCTQ in insulin group, the mean SSS before injection was $2.73 \pm 0.42$ while after injection was $1.35 \pm 0.26$. The mean FSS before injection was $2.32 \pm 0.71$ while after injection was $1.38 \pm 0.38$. As regard NCS in insulin group, the mean DML before injection was4.53 \pm 0.73 while after injection was $3.88 \pm 0.50$. The mean DSL before injection was $3.99 \pm 0.47$ while after injection was 3.747 \pm 0.31 .The mean MCV before injection was $51.97 \pm 5.68$ while after injection was 56.95 \pm 4.67 . The mean SCV before injection was $36.76 \pm 4.38$ while after injection was $43.24 \pm 3.28$.

\begin{tabular}{|c|c|c|c|c|c|c|}
\hline & & $\begin{array}{c}\text { Mea } \\
n\end{array}$ & \pm & $\begin{array}{l}\text { S. } \\
\text { D }\end{array}$ & t. test & $\begin{array}{c}\mathrm{p} . \\
\text { value }\end{array}$ \\
\hline \multirow{4}{*}{$\begin{array}{c}\text { BCT } \\
\text { Q } \\
\text { (SSS) } \\
\text { Insuli } \\
n\end{array}$} & Befor & & \pm & 0.4 & \multirow{4}{*}{$\begin{array}{c}197.39 \\
8\end{array}$} & \multirow{4}{*}{$\begin{array}{c}0.001 \\
*\end{array}$} \\
\hline & $\mathrm{e}$ & 2.73 & & 2 & & \\
\hline & \multirow[t]{2}{*}{ After } & & + & 0.2 & & \\
\hline & & 1.35 & & 6 & & \\
\hline \multirow{4}{*}{$\begin{array}{c}\text { BCT } \\
\text { Q } \\
\text { (FSS) } \\
\text { Insuli } \\
\text { n } \\
\end{array}$} & Befor & & & 0.7 & \multirow{4}{*}{34.049} & \multirow{4}{*}{$\begin{array}{c}0.001 \\
*\end{array}$} \\
\hline & $\mathrm{e}$ & 2.32 & \pm & 1 & & \\
\hline & \multirow[t]{2}{*}{ After } & & & 0.3 & & \\
\hline & & 1.38 & 1 & 8 & & \\
\hline \multirow{4}{*}{$\begin{array}{c}\text { DML } \\
\text { Insuli } \\
n\end{array}$} & Befor & & & 0.7 & \multirow{4}{*}{13.561} & \multirow{4}{*}{$\begin{array}{c}0.001 \\
*\end{array}$} \\
\hline & e & 4.53 & \pm & 3 & & \\
\hline & After & & \pm & 0.5 & & \\
\hline & & 3.88 & - & 0 & & \\
\hline \multirow{4}{*}{$\begin{array}{c}\text { DSL } \\
\text { Insuli } \\
n\end{array}$} & Befor & 300 & \pm & 0.4 & \multirow{3}{*}{21.624} & \multirow{3}{*}{$\begin{array}{c}0.001 \\
*\end{array}$} \\
\hline & After & 3.99 & & 0.3 & & \\
\hline & & 3.47 & & 1 & & \\
\hline & $\begin{array}{c}\text { Befor } \\
\mathrm{e}\end{array}$ & $\begin{array}{c}51.9 \\
7\end{array}$ & \pm & $\begin{array}{c}5.6 \\
8\end{array}$ & 11.460 & $\begin{array}{c}0.001 \\
*\end{array}$ \\
\hline
\end{tabular}




\begin{tabular}{|c|c|ccc|c|c|}
\hline MCV & After & & & & & \\
Insuli & & 56.9 & \pm & 4.6 & & \\
$\mathrm{n}$ & & 5 & & 7 & & \\
\hline \multirow{2}{*}{$\mathrm{SCV}$} & Befor & 36.7 & \pm & 4.3 & & \\
Insuli & $\mathrm{e}$ & 6 & \pm & 8 & \multirow{2}{*}{35.031} & 0.001 \\
\cline { 2 - 5 } $\mathrm{n}$ & After & $\begin{array}{c}43.2 \\
4\end{array}$ & \pm & $\begin{array}{c}3.2 \\
8\end{array}$ & & \\
\hline
\end{tabular}

Table 5, shows that there was statistical significant difference in BCTQ as regard SSS and FSS scores ( $p$-value $=0.001$ ) and also shows statistical significant difference in all of NCS parameters as regard DML, DSL, MCV and SCV (P-value $=0.001$ ), before and four weeks after second dose of insulin injection.

Table [6]:-Boston Carpal Tunnel Questionnaire and Electrophysiological finding after insulin and steroid injection.

As regard BCTQ after injection, the mean SSS in insulin group was $1.35 \pm 0.26$ while in steroid group was $1.92 \pm 0.44$. The mean FSS in insulin group was $1.38 \pm 0.38$ while in steroid group was $1.64 \pm 0.38$. As regard NCS after injection, the mean DML in insulin group was $3.88 \pm 0.50$ while in steroid group was $4.32 \pm 0.91$. The mean DSL insulin group was $3.47 \pm 0.31$ while in steroid group was $3.72 \pm 0.45$. The mean MCV in insulin group was $56.95 \pm 4.67$ while in steroid group was 54.26 \pm 4.47 . The mean SCV in insulin group was $43.24 \pm 3.28$ while in steroid group was $39.45 \pm 4.91$.

\begin{tabular}{|c|c|c|c|c|c|}
\hline & & Mean & \pm S. D & t. test & p. value \\
\hline \multirow{2}{*}{$\begin{array}{c}\text { BCTQ } \\
\text { (SSS) } \\
\text { (After) } \\
\end{array}$} & Insulin & 1.35 & \pm 0.26 & \multirow[b]{2}{*}{32.115} & \multirow[b]{2}{*}{$0.001 *$} \\
\hline & Steroid & 1.92 & \pm 0.44 & & \\
\hline \multirow{2}{*}{$\begin{array}{c}\text { BCTQ } \\
\text { (FSS) } \\
\text { (After) }\end{array}$} & Insulin & 1.38 & \pm 0.38 & \multirow[b]{2}{*}{5.994} & \multirow[b]{2}{*}{$0.018 *$} \\
\hline & Steroid & 1.64 & \pm 0.38 & & \\
\hline \multirow{2}{*}{$\begin{array}{l}\text { DML } \\
\text { (After) }\end{array}$} & Insulin & 3.88 & \pm 0.50 & \multirow{2}{*}{4.572} & \multirow{2}{*}{$0.038^{*}$} \\
\hline & Steroid & 4.32 & \pm 0.91 & & \\
\hline \multirow{2}{*}{$\begin{array}{l}\text { DSL } \\
\text { (After) }\end{array}$} & Insulin & 3.47 & \pm 0.31 & \multirow{2}{*}{5.236} & \multirow{2}{*}{$0.027 *$} \\
\hline & Steroid & 3.72 & \pm 0.45 & & \\
\hline \multirow{2}{*}{$\begin{array}{l}\text { MCV } \\
\text { (After) }\end{array}$} & Insulin & 56.95 & \pm 4.67 & \multirow{2}{*}{4.331} & \multirow{2}{*}{$0.043 *$} \\
\hline & Steroid & 54.26 & \pm 4.47 & & \\
\hline \multirow{2}{*}{$\begin{array}{c}\text { SCV } \\
\text { (After) }\end{array}$} & Insulin & 43.24 & \pm 3.28 & \multirow{2}{*}{10.326} & \multirow{2}{*}{$0.002 *$} \\
\hline & Steroid & 39.45 & \pm 4.91 & & \\
\hline
\end{tabular}

Table 6, shows that there was statistically significant difference between both groups in BCTQ as regard SSS and FSS scores (P-value $=0.001$ and $<0.05$ respectively) and also shows statistically significant difference between both groups in all of NCS parameters as regard DML, DSL, MCV and SCV (P-value <0.05), one month after steroid injection and four weeks after second dose of insulin injection.

\section{DISCUSSION}

This study was carried out to assess the effects of insulin versus corticosteroid local injections in diabetic patients with carpal tunnel syndrome.

It is believed that the most important factor in nerve compression injury is local pressure increase. Many studies supported that alteration of microvascular structure of the nerve occured in the early stages of the disease ${ }^{[15]}$. The arteries of diabetic people are already adversely affected by the diabetes itself. As a result, they may be more susceptible to minimal pressure increases in the carpal tunnel ${ }^{[16]}$.

It has been assumed that hyperglycemia induces metabolic derangement that directly affect Schwann cells (or myelin), nodes of Ranvier (or axons), and that hyperglycemia and metabolic derangement affect the structure and function of endoneurial microvessels, which could lead to a reduction in the endoneurial blood flow and oxygen tension, which then induce a breakdown in the blood-nerve-barrier inducing hypoxia or ischemia ${ }^{[8]}$ So, CTS can occur more commonly in diabetic patients.

The current study included 50 diabetic patients, 35(70\%) females and 15(30\%) males, in case of patients with bilateral CTS, the most symptomatic hand was selected, so the total hands in this study were 50 hands, with $28(56 \%)$ patients considered as mild and $22(44 \%)$ patients considered as moderate CTS. Their ages ranged from 30 to 60 years, with a mean of $46.14 \pm 8.30$ years. The diabetes disease duration ranged from 4 to 8 years, with a mean of 5.58 \pm 1.21 years. The symptoms of CTS duration ranged from 2 to 40 weeks, with a mean of $12.74 \pm 9.59$ weeks.

Local Steroid injections are known to be a safe and effective treatment for relieving symptoms in more than $75 \%$ of patients with CTS and have been superior to placebo in randomized clinical trials ${ }^{[17]}$.It has been reported that a single injection of steroids close to the carpal tunnel may result in long-term 
improvement and should be considered before surgical decompression. Due to its antiinflammatory effect, Steroids are effective at reducing swelling and so concerned as the cornerstone of conservative management in CTS ${ }^{[17]}$.

The current study showed that, local steroid injection (40 $\mathrm{mg}$ of triamcinolone acetonide), leads to significant improvement in not only the carpal tunnel Questionnaire as regard SSS score and FSS score but also, the nerve conduction study as regard DSL, SCV. On the other hand there was no significant improvement in DML and MCV. These results were in agreement with those obtained in the study conducted by Ozkul et al. who found that, steroid injection alone leads to significant improvement in SCV and global symptom scale (GSS) without significant improvement in DML in diabetic patients with a mild to moderate degree of CTS ${ }^{[16]}$.

The current study demonstrated that, local insulin injection (10 IU of NPH), produced a more statistically significant improvement in carpal tunnel Questionnaire as regard SSS score and FSS score, and also a more statistically significant improvement in nerve conduction study as regard DML, DSL, MCV and SCV, than did steroid injections. These results were in agreement with those obtained in the study conducted by Ashraf et al. who found a decrement in DML of the median nerve and an increment in the sensory nerve conduction velocity of the median nerve after local insulin injection in patients with type 2 diabetes mellitus with mild to moderate CTS. In addition, there was significant reduction in the mean symptom scores for pain, numbness, paresthesias, weakness/clumsiness and nocturnal awakening ${ }^{[18]}$.

Also in the study conducted by Ibrahim and Hussein, the results of current study were in agreement with their results which showed that insulin injections produced a more significant improvement in NCS (DML, DSL) and in BCTQ (FSS, SSS) Score than did steroid injections in non-diabetic patients with mild to moderate CTS ${ }^{[19]}$.

It has been suggested that insulin has an effect on nerve regeneration, similar to that of NGF. Both of these are thought to promote neuronal growth, regeneration, remylination and could be important in restoring nerve function following metabolic or vascular damage as neurotrophic factors ${ }^{[18]}$. Circulating NGF concentrations have been found to be reduced in diabetic patients with neuropathy to a degree which parallels the severity of the neuropathic damage ${ }^{[20]}$ and restoration by insulin can restores nerve function and regeneration ${ }^{[21]}$, so it has been thought that, local insulin injection in the CTS could lead to an improvement in the metabolism and structure of the median nerve as insulin has neutotrophic effects ${ }^{[16]}$.

In fact, it has been reported that insulin has anti-inflammatory effects and these antiinflammatory effects of insulin have been known since the discovery of the benefits of insulin therapy in systemic inflammatory responses to trauma or bacterial infection ${ }^{[22]}$. Currently, the effects are dependent upon its suppression of innate immune mechanisms and the suppression of transcription factors such as $\mathrm{NF} \kappa \mathrm{B}$ and Egr- ${ }^{[23]}$. In the study conducted by Abu-Zaid et al. reported that, local insulin injection is as effective as (or even better than) local steroid injection in management of CTS, as insulin has anti-inflammatory antiedematous effects ${ }^{[24]}$.

The current study showed that, the difference between results in both groups after the injection were statistically significant for insulin injection group in BCTQ as regard SSS and FSS scores and all parameters of NCS as regard DML, DSL, MCV and SCV. This results were disagreement with the results of the study conducted by Abu-Zaid et al. which showed that significant improvement in all clinical parameters and NCS in both insulin and steroid locally injected groups in non-diabetic patients with CTS with more improvement was noticed in insulin injection group as in the current study but the difference between results in both groups were statistically non-significant ${ }^{[24]}$.

This discrepancy between our results and those of Abu-Zaid et al. as regard significant difference between results in both insulin and steroid injection groups in the current study may be due to, the current study conducted on diabetic patients with CTS, whereas their study conducted on non-diabetic (idiopathic) patients with CTS which may be 
prove the role of insulin deficiency in the pathogenesis of the CTS in our patients with diabetes indicating that restoration of insulin locally injected around median nerve could lead to an improvement in the metabolism and structure of the nerve which affected by the insulin deficiency and hyperglycemia in diabetic patients and this may lead to us to speculate that local insulin injection in CTS is more effective in diabetic patients than in nondiabetic (idiopathic) patients, however further studies should be done comparing local insulin injection between diabetic and non-diabetic patient with CTS.

The choice of nature, method and dose of insulin injections in the current study was based on the previous published study in treating CTS in diabetic patients with local insulin injection ${ }^{[18]}$, where this dose has no effect on plasma glucose levels and gives significant benefit in restoring the median nerve function. in their study Ashraf et al. they found that plasma glucose and glycosylated hemoglobin (HbA1c) levels did not change during the course of study, so they reported that the effect of local insulin injection in improvement of CTS in diabetic patients was not due to changes of plasma glucose levels or due to glycemic control from the local insulin injection as the plasma glucose levels remained unchanged throughout the study ${ }^{[18]}$ but due to the local effect of insulin on the median nerve as neurotrophic factor.

\section{CONCLUSION}

In the current study, local insulin injections produce a more statistically significant improvement in all NCS parameters as regard decreased DML and DSL and increased MCV and SCV and also produce a more statistically significant improvement in Boston Carpal Tunnel Questionnaire (BCTQ) as regard reduced SSS and FSS scores four weeks after second dose of insulin injection than the local steroid injection produced one month after its injection in diabetic patients with CTS.

These data suggest that in diabetic patients with CTS local steroid injection produces significant improvement in the median nerve condition and local insulin injection produce a more significant improvement probably by inducing improvement in the metabolism and structural changes in the nerve through the local neurotrophic effect of insulin and through its local anti-inflammatory anti-edematous effects. Although these findings are promising, further studies with insulin are needed to verify its effectiveness as a treatment for CTS and other degenerative nerve diseases.

\section{RECOMMENDATIONS}

Diabetic patients with Carpal tunnel syndrome can be treated by local insulin injection in carpal canal which is effective as or more better than local steroid injection in the treatment of carpal tunnel syndrome as insulin has neurotrophic effect and anti-inflammatory anti- edematous effects.

Extended follow-up of our cases treated with local insulin injection is advised to show the long-term effect of local insulin injection treatment in diabetic patients with CTS. Furthermore, future short-term and long-term follow-up studies comparing insulin injection in diabetic and non-diabetic patients with CTS to show the effectiveness of local insulin injection in non-diabetic patients as in diabetic patients with CTS or not.

\section{REFERENCES}

1. Burton C, Chesterton LS, Davenport G(2014): Diagnosing and managing carpal tunnel syndrome in primary care. The British journal of general practice: the journal of the Royal College of General Practitioners, 64(622): 262-263.

2. Atroshi I, Englund M, Turkiewicz A et al. (2011): Incidence of physician-diagnosed carpal tunnel syndrome in the general population. Arch Intern Med. ,171(10):943943.

3. Stevens JC, Witt JC, Smith BE, Weaver AL (2001): The frequency of carpal tunnel syndrome in computer users at a medical facility. Neurology,56(11):1568-1570.

4. Dyck PJ, Thomas PK(2000): Diabetic Neuropathy. 2nd edn. Philadelphia: WB Saunders Company, 15(5): 379-379.

5. Katz JN, Simmons BP (2002): Clinical practice Carpal tunnel syndrome. N Engl J Med., 346(23):1807-1812.

6. Padua L, Coraci D, Erra C et al. (2016):Carpal tunnel syndrome: clinical features, diagnosis, and management. Lancet Neurology (Review), 15 (12): 1273-1284. 
7. Diao E, Shao F, Liebenberg E, Rempel D, Lotz JC (2005): Carpal tunnel pressure alters median nerve function in a dose-dependent manner: a rabbit model for carpal tunnel syndrome. J Orthop Res., 23 (1) 218-223.

8. MacDermid JC, Dohert T (2004): Clinical and electrodiagnostic testing of carpal tunnel syndrome a narrative review. J Orthop Sports Phys Ther. 34(10):565-588.

9. Ward JD, Tesfay S. (1997). Pathogenesis of neuropathy . In: Pickup JC, Williams G. editors. Textbook of diabetes. $2^{\text {nd }}$ ed. Malden, MA:Blackwell Science,2(49):1-49.19.

10. Apfel SC, Kessler JA, Adornato BT, Litchy WJ, Sanders C, Rask CA(1998): Recombinant human nerve growth factor in the treatment of diabetic polyneuropathy. Neurology, 51(3):695-702.

11. Singhal A, Cheng C, Sun H, Zochodne DW (1997): Near nerve local insulin prevent conduction slowing experimental diabetes . Brain Res.,763(94):209-214.

12. Bland JD(2000) : The value of the history in the diagnosis of carpal tunnel syndrome. $\mathbf{J}$ Hand Surg Br., 25(5):445-550.

13. Gay RE, Amadio PC, Johnson JC(2003): Comparative responsiveness of the Disabilities of the Arm, Shoulder and Hand, the Carpal Tunnel Questionnaire and the SF-36 to clinical change after carpal tunnel release. Journal of Hand Surgery, 28(2):250-254.

14. Boyer MI ( 2008 ): Corticosteroid injection for carpal tunnel syndrome. J Hand Surg Am.,33(8):1414-1416.

15. Alfonso C, Jann S, Massa R, Torreggiani A (2010): Diagnosis treatment and follow-up of the carpal tunnel syndrome: a review. Neurolog Sci., 31(3):243-252.

16. Ozkul Y ,Sabuncu, Yazgan P, Nazligul $Y$ (2001): Local insulin injection improves median nerve regeneration in NIDDM patient with carpal tunnel syndrome Eur J Neurol., 8(4):329-334.

17. Hagebeuk EE, Weerd AW (2004): Clinical and electrophysiological follow-up after local steroid injection in the carpal tunnel syndrome. Clin Neurophysiol.,115:1464-1468. 18. Ashraf A1, Moghtaderi AR, Yazdani AH, Mirshams S (2009): Evaluation of effectiveness of local insulin injection in noninsulin-dependent diabetic patient with carpal tunnel syndrome. Electromyogr Clin Neurophysiol., 49(4):161-166.

19. Ibrahim S, Hussein A (2016): New role for insulin injection in the treatment of idiopathic carpal tunnel syndrome . Egyptian Rheumatology \& Rehabilitation, 43 (4): 157162.

20. Luigi A, Maria L, Patrizia $B$ and Luigi $M$ (2012): Nerve growth factor: from the early discoveries to the potential clinical use. J Transl Med.,10: 239.

21. Kamiya H, Zhang W, Sima AA (2006): Degeneration of the Golgi and neuronal loss in dorsal root ganglia in diabetic BioBreeding/Worcester rats. Diabetologia ,49(11):2763-2774.

22. Jeschke MG, Einspanier R, Klein D, Jauch KW (2002): Insulin attenuates the systemic inflammatory response to thermal trauma. Mol Med.,8(8):443-450.

23. Qiang S, Jia L, and Feng G( 2014): New insights into insulin: The anti-inflammatory effect and its clinical relevance. World $\mathrm{J}$ Diabetes, 5(2): 89-96.

24. Abu-Zaid MH, El morsy S, Hablas SA (2017): Local steiod and insulin injection in management of carpal tunnel syndrome; a comparative study. BMJ journal, Annals of the Rheumatic Diseases (ARD), 76: 2. 